\title{
ABSTRACTS OF PAPERS
}

\section{SUBMITTED FOR PRESENTATION TO THE SOCIETY}

The following papers have been submitted to the Secretary and the Associate Secretaries of the Society for presentation at meetings of the Society. They are numbered serially throughout this volume. Cross references to them in the reports of the meetings will give the number of this volume, the number of this issue, and the serial number of the abstract.

\section{Algebra And Theory of Numbers}

\section{A. A. Albert: Division algebras over a function field.}

Let $D$ be a division algebra of degree $m$ over its centrum $K$ which is algebraic of finite degree over $L(x)$. The author studies the least degree $n$ of fields $W$ of finite degree over $L$ such that a composite of $K$ and $W$ splits $D$. Clearly $n \geqq m$ and one asks whether in general $n=m$. The answer is in the negative, since if $L$ is finite and $K=L(x)$, then $n$ may be any integer divisible by $m$ and such that every prime factor of $n$ divides $m$. The property $n>m$ is not a function of the finiteness of $L$ and this is indicated by proving it for $m=2$ and all fields $L$ such that there exist at least two inequivalent quadratic extensions of $L$. In particular when $L$ has characteristic two this result implies $n>m$ also for any $m=2^{e}$. The paper mentions finally an unpublished master's dissertation of Louis Gordon showing that if $m=4$ and $K$ is algebraic of finite degree over $L\left(x_{1}, \cdots, x_{r}\right)$ then $D$ may be non-cyclic if $r>2$ and the $x_{i}$ are independent indeterminates over $L$. However, it is proved that $D$ is necessarily cyclic for $r=2$. (Received July 14, 1941.)

\section{A. A. Albert: Non-associative algebras. I.}

The paper presents a new general theory of non-associative algebras. Any algebra $A$ is a linear space $L$ of finite order over a field. Multiplication in $A$ is then defined by a linear mapping $x \rightarrow R_{x}$ of $L$ on the space of right multiplications $R_{x}$ of $A$. A second algebra $A_{0}$ of the same order may be regarded as consisting of the same quantities as does $A$ but with multiplication defined by $x \rightarrow R_{x}^{(0)}$. Then $A$ and $A_{0}$ are called isotopes, or isotopic algebras, if there exist non-singular linear transformations $S, T, U$ on $L$ such that $R_{x}^{(0)}=S R_{y} U, y=x^{T}$. Isotopy is then a widening of the concept of equivalence except in the case of associative algebras with a unity quantity where the concepts coincide. This possible widening accounts at least partly for the inordinately large number of algebras of low orders. It is shown that there exist non-commutative isotopes with a unity quantity of commutative non-associative algebras with a unity quantity, and non-simple isotopes of simple algebras. Related topics inspired by isotopy and connections of the theories of associative algebras, Lie algebras and Jordan algebras are studied. (Received July 14, 1941.)

332. Reinhold Baer: Automorphism rings of primary abelian operator groups.

The representation of a ring as the ring of all the automorphisms of a primary abelian operator group expresses significant inner properties of this ring, since there 
exists essentially at most one such representation of a given ring. Thus it is the object of this investigation to expose invariant qualities of these automorphism rings. They are found to be peculiarities of their ideal-theory; and amongst these are listed a number which form a complete set of postulates for the class of all the automorphism rings of primary abelian operator groups, thus proving incidentally that these rings are completely determined by their ideal-theory. The properties of the ideals in these rings reflect so completely the structure of the underlying operator group that it is possible to prove the essential identity of the group of automorphisms of the ring and of the group of projectivities of the system of admissible subgroups of the underlying operator groups. (Received July 31,1941.)

\section{Richard Brauer: Investigations on group characters.}

The paper continues the previous work of C. Nesbitt and the author on modular characters of groups and their connection with the ordinary group characters. Let $G$ be a group of finite order $g=p^{a} g^{\prime}$ where $p$ is a prime which does not divide $g^{\prime}$. Let $F$ be an ordinary irreducible representation of $G$ whose degree is divisible by $p^{a-1}$. It is shown that the representations belonging to the block $B$ of $F(\bmod p)$ have the same property. The decomposition $(\bmod p)$ of the characters of $B$ can be described by a linear graph which is proved to be a tree. As an application, it can for instance be shown that a simple group of an order $4 p^{a} q^{b}(p, q$ primes, $a \leqq 2)$ must have order 60; a simple group of an order $3 p^{a} q^{b}$ ( $p, q$ primes, $\left.a \leqq 2\right)$ must have the order 60 or 168. (Received July 29, 1941.)

\section{Richard Brauer: On the indecomposable representations of alge-} bras.

The indecomposable representations $M$ of an algebra $A$ are studied, that is, the representations $M$ for which the underlying vector space is not the direct sum of two invariant subspaces. The necessary and sufficient conditions are obtained that $M$ is an end constituent of the regular representation of $A$. In addition, cases are studied in which $A$ has infinitely many non-equivalent indecomposable representations. (Received July 29,1941 .)

\section{R. H. Bruck: The number of absolute invariants of a tensor.}

From the method of L. P. Eisenhart (Continuous Groups of Transformations, pp. 62 and 20) it is deduced for the complex field that the number of functionally independent absolute invariants, under $G L(n)$, of a tensor $T_{(i)}^{(i)} \equiv T_{i_{1} j_{2} \ldots i_{q}}^{i_{1} i_{2} \ldots i_{p}}$ is equal to $N-Q$, where $N$ is the number of components of $T_{i}^{(i)}$ and $Q$ is a two-way rank of the tensor $\xi_{(j) \alpha}^{(i) \beta} \equiv\left[\delta_{i_{1}}^{\beta} T_{\alpha j_{2} \ldots i_{q}}^{i_{1} i_{2} \ldots i_{p}}+\cdots+\delta_{i q}^{\beta} T_{i_{1} i_{2} \ldots i_{\alpha}}^{i_{i_{2}} \ldots i_{p}}\right]-\left[\delta_{\alpha}^{i_{1}} T_{j_{1} i_{2} \ldots i_{p}}^{\beta_{2} \ldots}+\cdots+\delta_{\alpha}^{i_{p}} T_{i_{1} i_{2} \ldots i_{q}}^{i_{1} i_{2} \ldots \beta}\right]$. The problem of finding $Q$ reduces to the purely algebraic one of determining the number $n^{2}-Q$ of linearly independent tensors $F_{\beta}^{\alpha}$ satisfying $\left({ }^{*}\right) \xi_{(i) \alpha}^{(i) \beta} F_{\beta}^{\alpha}=0$. The equations $\left(^{*}\right)$ may be replaced by the sets of simultaneous equations obtained by substituting in turn for $T_{(i)}^{(i)}$ each of its symmetrized parts (T. L. Wade, American Journal of Mathematics, vol. 63 (1941), pp. 645-657). The method is applied to various tensors. (Received July 30, 1941.)

\section{R. H. Bruck and T. L. Wade: On a tensor algebra of Cramlet.}

In this paper some concepts and methods of the general theory of linear associative algebras of tensors developed recently by the authors (Bisymmetric tensor algebra, Parts I and II, to appear in American Journal of Mathematics; see also this Bulletin, 
abstracts 47-5-184 and 47-5-185) are extended. This extended form of general tensor algebra is related to a particular tensor algebra considered by C. M. Cramlet (Annals of Mathematics, (2), vol. 31 (1930), pp. 134-150) in connection with differential forms. (Received July $26,1941$.

\section{H. H. Campaigne: An example of a cogroup.}

An example is given which answers a question of J. E. Eaton (Theory of cogroups, Duke Mathematical Journal, vol. 6 (1940), pp. 101-197). It is shown that this cogroup cannot be derived from any group by any conjugation. (Received July 25,1941 .)

\section{A. D. Campbell: On the application of barycentric coordinates} to lattice theory.

One can label the elements of any specific lattice with barycentric coordinates in such a way that the least upper bound and the greatest lower bound of any two elements can be read off from the subscripts of these barycentric coordinates. Also by using abstract sets for these subscripts (instead of integers) many old and new results in lattice theory can be proved quickly. If $P_{\alpha}$ is one element of a lattice and $P_{\beta}$ another element then $P_{\alpha} \cap P_{\beta}=P_{\alpha \cdot \beta}$, where $\alpha \cdot \beta$ is the meet of the sets $\alpha$ and $\beta$. Also $P_{\alpha} \cup P_{\beta}=P_{\alpha, \beta, a_{\alpha}, \beta}$, where $\alpha, \beta, a_{\alpha, \beta}$ means the join of the sets $\alpha, \beta$ and $a_{\alpha, \beta}$ and where $a_{\alpha, \beta}$ is a set whose presence in the subscript is caused by the presence of the join $\alpha, \beta$. The necessary and sufficient condition for a lattice to be modular is: If $P_{\alpha}, P_{\beta}, P_{\gamma}$ are any three elements of this lattice such that $\alpha \leqq \gamma$, then $\gamma \cdot\left(a_{\alpha, \beta}-a_{\alpha, \beta, \gamma}\right)=0$. The necessary and sufficient conditions for a lattice to be distributive reduce to $\alpha \cdot\left(a_{\beta, \gamma}-a_{\alpha \cdot \beta, \alpha \cdot \gamma}\right)=0$, where $P_{\alpha}, P_{\beta}, P_{\gamma}$ are any three elements of the lattice. These barycentric coordinates seem to offer great possibilities in the handling of lattice theory. (Received July 2, 1941.)

339. P. W. Carruth: Arithmetic of ordinals with applications to the theory of ordered abelian groups.

Hessenberg has defined a "natural sum" of ordinals, and Hausdorff has defined a "natural product" of ordinals. This paper gives new characterizations of these operations. It is shown that Hessenberg's natural sum of two ordinals $\alpha$ and $\beta$ is the best bound for the order type of the join of two well-ordered subsets, of respective types $\alpha$ and $\beta$, of an ordered set. Hausdorff's natural product of two ordinals $\alpha$ and $\beta$ is shown to be the best bound for the order type of a certain rectangular array of ordered elements that has " $\alpha$ rows" and " $\beta$ columns." Another application of Hausdorff's natural product is given in determining a bound for the order type of the semi-group generated by a well-ordered subset of positive elements of an ordered abelian group. (Received June 6, 1941.)

\section{R. J. Duffin and R. S. Pate: Generalized groups and the Jordan- Hölder theorem.}

Generalized groups (hypergroups, quasigroups, groupoids, and so on) arise by removing some of the group postulates. For a commutative group the Jordan-Hölder theorem concerns all subgroups, but if the commutative postulate is removed the theorem concerns only subgroups having a commutative behavior. Likewise the generalized groups have a Jordan-Hölder theorem provided every postulate removed from the group as a whole is transferred in a suitable manner to the "subgroups" con- 
cerned. Most generally the "group" becomes nothing more or less than a non-commutative, non-associative, multiple valued product. It is convenient to restore the unique product by adjoining subsets of elements to the original elements. The generalized group becomes then a partially ordered set each of whose elements effects by the product operation an order preserving automorphism. It is to be emphasized that the Jordan-Hölder theorem for such a system is more than an analogy with the theorem for groups; indeed the theorem for groups is a direct consequence of the generalized theorem. For a commutative group, what is meant by the Jordan-Hölder theorem is unambiguous, but for a non-commutative group the theorem applies to both principal series and to composition series. (Received July 30,1941.)

\section{Fritz Herzog: On an application of a theorem by Burnside.}

The theorem, referred to in the title, states that if a Sylow subgroup of a finite group $G$ is contained in the center of its normalizer, then $G$ contains a normal subgroup the index of which is the order of that Sylow subgroup. This theorem is applied to obtain the result that a group of order $g$ all of whose Sylow subgroups are abelian must be abelian itself if certain number theoretical relations between the prime factors of $g$ are satisfied. As a conclusion from the latter theorem, the totality of all positive integers $g$ is determined which have the property that all groups of order $g$ are abelian. (Received July 30, 1941.)

\section{S. A. Jennings: Central chains of ideals in an associative ring.}

Let $A, B$ be any two (not necessarily distinct) ideals of an associative ring $R$; then the ideal $A \circ B$ is defined to be the ideal generated by all elements of the form $a b-b a$, where $a \in A, b \in B$. A chain of ideals of $R, R=M_{1} \supseteq M_{2} \supseteq \cdots \supseteq M_{m} \supseteq M_{m+1}=0$ terminating with the 0 -ideal is a central chain if $M_{\rho} \circ R \subseteq M_{\rho+1}, \rho=1,2, \cdots$. A ring with a central chain is said to have finite class. In particular every nilpotent ring is of finite class. For such a ring the lower central chain is defined by setting $R=H_{1}$, $H_{\rho}=H_{\rho-1} \circ R$. Properties of this chain analogous to those of the lower central series of a nilpotent group are obtained. Also the upper central chain is defined, corresponding to the upper central series in a group. A ring is said to be solvable if the chain defined by setting $R=R^{(0)}$ and $R^{(n)}=R^{(n-1)} \circ R^{(n-1)}$ terminates with the 0 -ideal. Some criteria for a ring to have finite class and for solvability are obtained and some applications considered. (Received July 25, 1941.)

\section{B. W. Jones: On an extension of a theorem of Witt.}

Ernst Witt (Journal für Mathematik und Physik, vol. 176 (1937), pp. 31-48) proved that if, in any algebraic field $F$ of characteristic $\neq 2$, there is a transformation $\mathfrak{T}$ in $F$ taking the vector space $\mathfrak{S}_{1}+\widetilde{S}_{2}$ into $\widetilde{S}_{1}+\widetilde{S}_{3}$, where $\widetilde{S}_{3}$ and $\widetilde{S}_{2}$ are vector spaces in $F$ orthogonal to $\mathfrak{S}_{1}$, then there is such a transformation taking $\widetilde{S}_{2}$ into $\widetilde{S}_{3}$. This paper proves the corresponding result for a ring $R_{p}$ of $p$-adic integers with $p$ odd, where not only $\mathfrak{I}$ but its inverse is in $R_{p}$. The same result holds for any $\mathfrak{P}$-adic ring over an algebraic field where $\mathfrak{P}$ is a prime ideal relatively prime to 2 . These results may also be stated in terms of quadratic forms. (Received July 28, 1941.)

\section{G. K. Kalisch: On Jordan algebras.}

An algebra $A$ over a field $F$ will be termed a Jordan algebra in case there exists an associative algebra $B$ over $F$ with an involutorial anti-automorphism $J$ such that $A$ is isomorphic to the algebra of the $J$-symmetric elements of $B$. Let $C$ be a distributive 
algebra of finite order over a field $F$ contained in the field $R$ of all real numbers. Suppose that the scalar extension $C \times R$ is the Jordan algebra defined as above for $B$ either (1) the algebra of all $n$-rowed real matrices, (2) the algebra of all $n$-rowed complex matrices, (3) the algebra of all $n$-rowed matrices with quaternionic elements. Then it is proved that in all three cases the given algebra $C$ over $F$ is a Jordan algebra, in cases (1) and (3) for a normal simple algebra $S$ over $F$ such that $S \times R=B$, and in case (2), for a simple algebra $S$ over $F$ with centrum $K=F(q)$ where $q^{2}=a<0$ in $F$, $J$ is an involutorial anti-automorphism of the second kind, and $S \times R=B$. Finally a classification of such algebras is given by means of congruent matrices as in the Lie algebra theory of N. Jacobson. (Received July 21, 1941.)

\section{Morris Marden: The zeros of certain composite polynomials.}

In this article the relative location of the zeros of the polynomials $A(z)=\sum_{k=0}^{m} a_{k} z^{k}$, $B(z)=\sum_{k=0}^{n} b_{k} z^{k}, C(z)=\sum_{k=0}^{m} a_{k} B(k) z^{k}$ is discussed. It is proved, for example, that, if all the zeros of $A(z)$ lie in the ring $0 \leqq r_{1} \leqq|z| \leqq r_{2} \leqq \infty$ and if all the zeros of $B(z)$ lie in the connected region bounded by two circles $|z|=\lambda_{1}|z-m|$ and $|z|=\lambda_{2}|z-m|$, then all the zeros of $C(z)$ lie in the ring $R_{1} \leqq|z| \leqq R_{2}$, where $R_{1}=r_{1}|B(0) / B(m)|, r_{1} \lambda_{1}^{n}$, or $r_{1}$ and $R_{2}=r_{2}, r_{2} \lambda_{2}^{n}$, or $r_{2}|B(0) / B(m)|$ according as $0 \leqq \lambda_{1} \leqq \lambda_{2} \leqq 1,0 \leqq \lambda_{1} \leqq 1 \leqq \lambda_{2}$, or $1 \leqq \lambda_{1} \leqq \lambda_{2}$. A similar theorem is proved for the case that all the zeros of $A(z)$ lie in a half-plane. The theorems found here are generalizations of theorems due to Laguerre, Pólya, and Weisner. The results are also extended to entire functions of genre 0 or 1 . (Received July 29, 1941.)

\section{Deane Montgomery and Leo Zippin: A theorem on Lie groups.}

This note gives and proves a partial generalization of the following theorem: Let $G$ be a Lie group and let $G^{*}$ be a compact subgroup of $G$. Then there exists an open set $O$ including $G^{*}$ which has the property that if $H$ is a subgroup of $G$ which is in $O$, then there exists an element $g$ in $G$ such that $g^{-1} H g$ is in $G^{*}$. This can be regarded as a generalization of the theorem that a Lie group has no subgroup near the identity except the identity itself. (Received July $3,1941$. )

347. D. C. Murdoch: Automorphisms and quasi-groups. Preliminary report.

An abelian quasi-group is one which satisfies the law $(a b)(c d)=(a c)(b d)$. It is said to be right nilpotent if the series of successive right unit subquasi-groups terminates in an idempotent element. Every right nilpotent quasi-group is a direct product of cyclic quasi-groups. If it is both left and right nilpotent then there exists a composition series in which successive quotient groups are all associative, and therefore ordinary groups. These and other results may be translated into statements concerning automorphisms of abelian groups. (Received July 28, 1941.)

\section{Ivan Niven: The mth roots of a quaternion.}

Any quaternion which is not a real number has exactly $m$ distinct $m$ th roots. These are exhibited in terms of the quaternion and the complex roots of the real quadratic equation satisfied by the quaternion (involving the trace and norm as coefficients). A real number has infinitely many $m$ th roots in quaternions if $m \geqq 3$; if $m=2$, there are infinitely many square roots or just two according as the real number is negative or not. The method depends upon the establishing of a real equation satisfied by the quaternion; such an equation must be divisible by the quadratic referred to above. (Received July 29, 1941.) 


\section{Rufus Oldenburger: The factorization of polynomials.}

It is proved that a polynomial $f$ in one variable factors for a field $K$ into a product $g h$ of relatively prime polynomials $g$ and $h$ if and only if a certain polynomial $F$ associated with $f$ can be written for $K$ as a sum $G+H$, where $G$ and $H$ are related to $g$ and $h$ respectively in the same way as $F$ is to $f$. The sum $G+H$ corresponds uniquely to the product $g h$. The reducibility of a polynomial in one variable, with respect to a field $K$, is thus transformed into the problem of splitting a second polynomial into a certain sum of polynomials. Using these results it is shown that a binary form $B$ with coefficients in a field $K$ can be written in a finite number of ways as a sum $U+M$ of binary forms with coefficients in $K$, where if $K$ is extended to a closed field $K^{\prime}$, the form $U$ has a unique minimal representation with respect to $K^{\prime}$, and $M$ is a form of degree $n$, whose minimal number cannot be increased by the addition of a term $a L^{n}, L$ linear, to $M$. The splitting of $B$ into $U+M$ is invariant in the usual sense. (Received July 28, 1941.)

\section{Gordon Pall: Quaternions and sums of three squares.}

U. V. Linnik (Bulletin of the Academy of Sciences of the USSR, Mathematics Series, vol. 4 (1940), pp. 363-402) gives the following result: Let $p$ be an odd prime, $m$ large, $m \neq 4 k$ or $8 k+7,(-m \mid p)=1, x_{0}^{2} \equiv-m(\bmod p)$. Then as $x$ ranges over all proper pure quaternions $i_{1} x_{1}+i_{2} x_{2}+i_{3} x_{3}$ of norm $m$, every quaternion of norm $p$ occurs as a leftdivisor of $x_{0}+x$. His proof has the following serious error, which is adjusted in this article: He supposes that the number of representations as a sum of three squares of a binary quadratic form of determinant $D$ does not exceed $c_{\epsilon} D^{\epsilon}$. (Received June 5, 1941.)

\section{H. A. Simmons: Classes of maximum numbers associated with two symmetric equations in $n$ reciprocals.}

The terminology used in stating the results here is that which was employed in the author's article with W. E. Block (Duke Mathematical Journal, vol. 2, p. 317). The first equation considered is $\sum_{n, n-1}(1 / x)+\sum_{i=1}^{m} a_{i}(\pi(x))^{-i}=b / a,(a=(c+1) b-1$, $\left.\pi(x)=x_{1} x_{2} \cdots x_{n}\right)$, in which $b, c$, and $m$ are arbitrary positive integers, $n>1$, and the $a_{i}$ are any non-negative real numbers. The second equation is $\sum_{n, n-2}(1 / x)$ $+\lambda \sum_{n, n-1}(1 / x)+\mu \sum_{n, n}(1 / x)=b / a$, in which $a$ and $b$ are arbitrary positive integers, $n>2, \lambda$ is a non-negative integer, and $\mu$ is a positive integer. The Kellogg-solution of each of these equations is shown to have the remarkable properties. These results are obtained through several innovations of the previous methods of identifying maximum numbers. A new lemma and a new term, "s-set," play important roles relative to each equation. Another element of newness in the argument relative to the second equation is the introduction for the sought maximum number of an upper bound analogous to an upper bound that Curtiss (American Mathematical Monthly, vol. 29, pp. 380-387) used in his solution of Kellogg's Diophantine problem. (Received July $28,1941$.

\section{Olga Taussky and Ernest Best: A class of groups.}

A $t$-group $G$ is a group in which every subgroup $g_{2}$ self-conjugate in a subgroup $g_{1}$, itself self-conjugate in $G$, is self-conjugate in $G$. A $t$-group can also be defined as a group for which composition and chief series coincide. Abelian, hamiltonian and simple 
groups are trivial examples. In this paper general properties of $t$-groups and a large class of non-trivial $t$-groups are investigated. This class includes those groups which have cyclic Sylow subgroups, in particular all groups with square-free order. (Received July 10,1941.)

\section{R. M. Thrall: On symmetrized Kronecker powers and Lie repre-} sentations of the full linear group.

Let $\Omega$ be the free Lie ring with $n$ generators and denote by $\ell^{i}$ the ideal of $R$ genererated by all monomials of degree $i$ in the generators. The factor ring $L^{i} / L^{i+1}$ serves as representation space for a representation $A \rightarrow L_{i}(A)$, called the $i$ th Lie representation, of the full linear group, $G L(n)$. A recursion formula is obtained for trace $L_{i}(A)$ which expresses it as a sum of terms each the trace of a Kronecker direct product of symmetrized Kronecker powers of $L_{j}(A)$ with $j<i$. The first part of the paper is therefore devoted to a study of the analysis of the symmetrized Kronecker powers of an arbitrary representation of $G L(n)$. In the final sections of the paper the specific analysis of $L_{i}(A)$ is given for $i \leqq 10$. The decomposition of the various Lie representations is closely associated with the determination of characteristic ideals of $\mathbb{R}$. (Received July 23, 1941.)

\section{T. L. Wade: Euclidean concomitants of the ternary cubic.}

The results of C. M. Cramlet (this Bulletin, vol. 34 (1928), pp. 334-342) and of the writer (this Bulletin, vol. 47 (1941), pp. 475-478) which relate tensor algebra and invariant theory are used in the construction, reduction, and investigation of the geometric interpretations of euclidean concomitants of the ternary cubic $T_{a b c} X^{a} X^{b} X^{c}$. Here $a, b, c=1,2,3$ and $T_{a b c}$ is symmetric. (Received July 19, 1941.)

\section{Louis Weisner: Roots of certain classes of polynomials.}

From two polynomials $\phi(z)$ and $f(z)=\sum_{k=0}^{n} c_{k} z^{k}$, form the polynomial $g(z)$ $=\sum c_{k} \phi(2 k-n) z^{k}$. The author proves: (1) If the roots of $\phi(z)$ lie on the axis of pure imaginaries and the roots of $f(z)$ in the ring $r_{1} \leqq|z| \leqq r_{2}$, then the roots of $g(z)$ lie in this ring. (2) If the roots of $\phi(z)$ lie in the half-plane $R z \leqq 0$, and the roots of $f(z)$ in the circular region $|z| \leqq r$, then the roots of $g(z)$ lie in this circular region. (Received June 20, 1941.)

\section{L. R. Wilcox: Complementation and modularity in lattices.}

Let $L$ be a lattice with 0,1 . If $a, b \in L$, then $c$ is a left (right) complement within $b$ of $a$ in $a+b$ if $c \leqq b, c+a=b+a$, and $(c, a) \perp((a, c) \perp)$, where $(c, a) \perp$ means $c a=0$ together with $(d+c) a=d+c a$ for every $d \leqq a$. If for every $a, b \in L$ there exists a left (right) complement within $b$ of $a$ in $a+b$, then $L$ is said to be left (right) complemented. Properties of these notions are studied and some of the results are the following. (1) If $(a, b)$ is a modular pair, then $c$ is a right complement within $b$ of $a$ in $a+b$ if and only if $c$ is a right complement within $b$ of $a b$ in $b$. (2) If $L$ is left complemented, then $L$ is right complemented, the converse of (1) holds, and $L$ is symmetric in the sense that the relation of modularity is symmetric. (3) If $L$ is left complemented, and if $S$ is the set of all products $b c$ with $(b, c)$ not modular, then $x \in S, y \leqq x$ implies $y \in S$. A class of examples of left complemented lattices arising as subsets of a modular lattice is given, and the foundations of an extension theory for left complemented lattices are laid. (Received July 30,1941.) 


\section{A. T. Brauer: On the least quadratic non-residue.}

Let $p$ be a prime and $d$ the least quadratic non-residue $(\bmod p)$. The best estimate for $d$ was obtained by Vinogradow by analytical methods. This estimate, however, is valid only for sufficiently large primes. It is of interest to obtain such estimates also by elementary methods, in particular if these estimates are valid for all primes or for all primes of a fixed set. Gauss's first proof of the law of reciprocity gives such a bound for the primes of the form $8 n+1$. His result was improved and generalized by Schur, Nagell, and the author. In this paper these results will be improved still further. Moreover the author obtains bounds for the least quadratic non-residue which is not divisible by $d$, and bounds for the number $R$ of quadratic residues and for the number $N$ of quadratic non-residues which are less than $p^{1 / 2}$. This latter result improves theorems of Pólya, Schur, and Landau for a special case. (Received July 29, 1941.)

\section{Leonard Carlitz: Note on generalized Bernoulli and Euler num-} bers.

H. S. Vandiver (Proceedings of the National Academy of Sciences, vol. 23.(1937), pp. 555-559) has introduced certain numbers that he calls generalized Bernoulli numbers of the $r$ th order. For the first order they are defined by $b_{n}(m, k)=(m b+k)^{n}$. For these numbers Vandiver proves a generalized Staudt-Clausen theorem. 'The present note contains a simple proof of this theorem using Lucas' method. By means of this method a theorem of the same type is proved for the generalized number $\left(m_{1} b+k_{1}\right)^{n_{1}} \cdots\left(m_{t} b+k_{t}\right)^{n_{t}}$ which is suggested by certain Bernoulli polynomials in several variables. For the corresponding Euler numbers congruences of Kummer's type are derived. (Received July $30,1941$.

\section{Leonard Carlitz: The coefficients of the reciprocal of a series.}

A. Hurwitz (Mathematische Annalen, vol. 51 (1899), pp. 196-226 and Mathematische Werke, vol. 2, pp. 342-373) defined rational numbers $E_{m}$ by means of $\wp(u)=1 / u^{2}+\sum_{1}^{\infty}\left(2^{4 m} E_{m} / 4 m\right)\left(u^{4 m-2} /(4 m-2) !\right)$, where $\wp(u)$ is the Weierstrass function for the lemniscate case. Making use of the complex multiplication of $\wp(u)$ he proved a theorem of the Staudt-Clausen type for $E_{m}$. The present paper is concerned with series of the form $f(u)=\sum_{1}^{\infty} a_{m} u_{m} / m$ !, where $a_{m}$ is integral. Let the inverse of $f(u)$ be $\lambda(u)=\sum e_{m} u_{m} / m$ !, and assume that $e_{m}$ is a multiple of $(m-1)$ ! Then theorems of the Staudt-Clausen type are proved for the coefficients in the expansion of $u / f(u)$ and $u^{2} / f^{2}(u)$. The latter includes Hurwitz' theorem (except for the partial fraction corresponding to the prime 2). The method is entirely elementary and makes use of certain general ideas on series of the form $f(u)$ due to Hurwitz. (Received July 30,1941 .)

360. J. M. Dobbie: A certain method in additive number theory. Preliminary report.

Ratio difference equations are used to generate identities involving sums of squares of integers. The method is that used by Carmichael in his lectures and follows a method of Cauchy (Oeuvres, series 1, vol. 8, pp. 42-115). It is related to the well known theta function method of Jacobi. Starting with the equation $u(x)=(1-t x) u(t x)$, $0<|t|<1$, the function $\prod_{\nu=1}^{\infty}\left[\left(1-t^{\nu}\right)^{2}(1-x) /\left(1+t^{\nu}\right)^{2}(1+x)\right] \prod_{v=1}^{\infty}\left(1-t^{\nu} x\right)\left(1-t^{\nu} x^{-1}\right)$ $\div\left(1+t^{\nu} x\right)\left(1+t^{\nu} x^{-1}\right)$ is introduced and is expanded into a series about its poles. Special cases of the resulting identity are the classic identities for the forms $a^{2}+b^{2}$, $a^{2}+2 b^{2}, a^{2}+3 b^{2}$. By raising $G(x, t)$ to powers, identities for treating the forms 
$\sum_{i=1}^{n} a_{i}^{2}+r \sum_{i=1}^{n} b_{i}^{2}$ ( $n$ any positive integer, $r=1,2,3$ ) are obtained. Other values of $r$ can be treated by extending the function $G(x, t)$. This has been done for $r=4,5,7$, but the expansion for general $r$ has not been obtained. The method also has been used to treat certain related forms. (Received July 29, 1941.)

\section{R. D. James: On the sieve method of Viggo Brun.}

A. A. Buchstab (Matematicheskii Sbornik, vol. 46 (1938), pp. 375-387, and Comptes Rendus de l'Académie des Sciences, URSS, vol. 29 (1940), pp. 544-548) has made improvements in the sieve method and so obtained more precise results. It is the purpose of this paper to point out that his method applies equally well to any infinite set of primes satisfying certain conditions. By applying the method to the set of primes congruent to $3(\bmod 4)$, for example, it is shown that there is an infinite number of integer pairs $n, n+4$, each having exactly one prime factor congruent to 3 (mod 4). (Received July 28, 1941.)

\section{Ivan Niven: Quadratic diophantine equations in the rational and quadratic fields.}

Consider the general quadratic equation in two variables with rational integral coefficients, with non-negative discriminant (this restriction being imposed in order that the graph of the equation be not restricted to a finite region of the plane). Then one solution in integers of this equation implies infinitely many such solutions if and only if the graph of the equation is not an hyperbola with rational asymptotes or a pair of essentially irrational straight lines. If the coefficients of the equation are integers of a real quadratic field, one solution in integers of the field implies an infinite number if and only if the equation does not represent one of the following: no locus, a point, a pair of straight lines with coefficients essentially outside the quadratic field, or an ellipse with totally negative discriminant. A similar theorem is obtained for imaginary quadratic fields, the results being similar to the rational case. The principal parts of these theorems are proved by use of criteria for the solvability of the Pell equation in rational and quadratic fields; the criteria for quadratic fields are determined with the help of a theorem of Hilbert on the units of algebraic fields. (Received July 28,1941 .)

\section{Analysis}

\section{R. P. Agnew: Analytic extension by Hausdorff methods.}

Let $\chi(t), 0 \leqq t \leqq 1$, be a mass function which generates a regular Hausdorff method of summability $H(\chi)$. Let the number $r$, which is the greatest lower bound of numbers $\rho$ such that $\chi(t)$ is constant over $\rho \leqq t \leqq 1$, be called the order of $H(\chi)$. Let $\sum c_{n} z^{n}$ be a power series with a positive finite radius of convergence. Corresponding to each vertex $\zeta$ of the Mittag-Leffler star, let $B(r, \zeta)$ denote the set of points $z$ for which $\left|z-\left(1-r^{-1}\right) \zeta\right|<r^{-1}|\zeta|$. Let $B(r)$ denote the set of inner points of the intersection of the sets $B(r, \zeta)$. It is shown that $\sum c_{n} z^{n}$ is uniformly summable $H(\chi)$ over each closed subset $F$ of $B(r)$. The geometric series $\sum z^{n}$ is non-summable $H(\chi)$ at each point exterior to the closure of $B(r)$. A series $\sum u_{n}$ is called summable $\mathcal{H}$ to $\sigma$ if there is at least one regular method $H(\chi)$ which evaluates $\sum u_{n}$ to $\sigma$. Some properties of the method $\mathcal{H}$ are obtained. The existence of series with bounded partial sums which are not summable $\mathfrak{H C}$ is implied by the following Tauberian gap theorem. If $0<n_{1}<n_{2}<\cdots$, if $n_{p+1} / n_{p} \rightarrow \infty$ as $p \rightarrow \infty$, if $u_{n}=0$ when $n \neq n_{1}, n_{2}, \cdots$, and if $\sum u_{n}$ is summable $\mathfrak{H C}$, then $\sum u_{n}$ is convergent. (Received June 23, 1941.) 